\title{
Topological solitons in an DNA molecule spin chain
}

\begin{abstract}
When spins are described in the Lie algebra of $S U(2)$ the linking number can be ascertained from the Chern-Simons topology associated with the spin system. The elastic energy associated with bending (curvature) and twisting (torsion) can be formulated in terms of $S U(2)$ gauge fields. It is shown that bend and twist are not two separate entities but one depends on the other. This formalism helps us to depict the thermodynamic entropy as entanglement entropy and the entanglement of spin can be used as a resource for genetic information. This implies that the transcription of genetic information can be considered in the framework of quantum information theory. The present analysis also suggests that DNA loops in a super coil appear as topological solitons (skyrmions).
\end{abstract}

Volume 2 Issue 6 - 2018

\section{Subhamoy Singha Roy}

Department of Physics, JIS College of Engineering,West Bengal University of Technology, India

\author{
Correspondence: Subhamoy Singha Roy, Department of \\ Physics, JIS College of Engineering (Autonomous), West Bengal \\ University of Technology, Kalyani, Nadia, 74I235, India, \\ Email ssray.science@gmail.com
}

Received: January 23, 2018 | Published: November 28, 2018

Keywords: DNA super coil, linking number, elastic forces, entanglement entropy, soliton

\section{Introduction}

It is now well known that DNA can be regarded as a physical elastic object in a viscous environment. Two strands of double helix are antiparallel and two polynucleotide chains are coiled about the same axis such that B-DNA (Z-DNA) has right-handed (left -handed) helical sense. The existence of super coiled DNA has been confirmed in experiments long ago and it was found that in vivo chromosomal DNA molecules contain topological domains along which super coiling canoccue. ${ }^{1-3}$ DNA molecules from prokaryotes (cells without nuclear membranes) oft en adopt the interwound structures which are called plectonemic" super coils. In eukaryotes (cells with nuclei and other organelles with their own internal membranes) chromosomal DNA molecules are also known to be organized into topological independent loops..$^{2-5}$ Statistical mechanics of super coiled DNA has been studied by several authors. ${ }^{6}$

The experiments of Boles et al. ${ }^{7}$ suggest that thermal fluctuations determine the structure of super coils. Experiments of Bednar et.al. ${ }^{8}$ indicated that DNA-DNA attraction may compete with fluctuation entropy. In this note we shall study different statistical mechanical aspects of DNA super coils by taking into consideration that a DNA super coil can be viewed as a chain of spin system. In fact as two polynucleotide chains are coiled about the same axis with a specific helical sense in a DNA molecule, we may visualize it such that a spin with a specific orientation is inserted on the axis in the coil such that two adjacent coils have opposite orientations of the spin. This follows from the fact that with each turn two strands move in the opposite side of the axis and so the spin orientations assigned for two adjacent coils should be opposite to each other. In view of this a DNA super coil may be considered to represent an antiferromagnetic chain of spins located on the axis of the super coil. We shall study the topological properties as well as the elastic and thermodynamical properties of a DNA super coil from an analysis of this spin system .Indeed, the topological property such as the linking number can be derived from the Chern- Simons topology associated with a quantum spin., ${ }^{9,10}$ The elastic properties such as bending (curvature) and twisting (torsion) can be formulated in terms of gauge fields when spins are transcribed as gauge currents. ${ }^{9,10}$ The added bonus in this formulation is that it directly indicates the interdependence of bending and twisting. The thermo dynamical entropy associated with a DNA super coil appears here as the entanglement entropy of the antiferromagnetic spin chain.
It may be pointed out that entanglement entropy of a DNA super coil essentially represents the total amount of information that can be transcribed. Thus we arrive at a quantitative description of the genetic information contained in the super coil and the process of genetic information transcription can be visualized in the framework of quantum information theory. Also we shall show that DNA loops in the super coil appear as topological objects like solitons (skyrmions).

In Sec. 2 we shall formulate DNA super coil as a spin chain. In Sec. 3 we shall study the topological property of a DNA super coil from the perspective of such a spin chain. In Sec. 4 we shall formulate the elastic properties and in see. 5 we shall compute the entanglement entropy of the super coil. In Sec.6 we shall show that DNA loops can be viewed as topological objects like solitons in this formulism.

\section{DNA as a spin chain}

We consider that as two polynucleotide chains are coiled about the same axis with a specific helical sense in a DNA molecule, this can be viewed as if a spin with a specific orientation is inserted on the axis of the coil such that two adjacent coils have opposite orientations of the spin. In fact with each turn two strands move in the opposite side of the axis and so the spin orientation assigned for the two adjacent coils should be opposite to each other. Thus a DNA super coil may be viewed as a long chain of an antiferromagnetic spin system when the spin is considered to be located on the axis of the super coil. A unit vector depicting the tangent $\vec{t}=\partial_{s} \vec{r}$ where $\vec{r}(s)$ is a space curve parameterized by the arc length $S$ can be associated with a spin vector when the spin is located at the spatial point $X$ on the axis. A spin vector in the Lie algebra of $S U(2)$ representation can be constructed with bosonic or fermionic oscillators. We write the spin vector $\vec{S}(x)$ as

$$
\vec{S}(x)=\psi_{\alpha}^{\mathrm{t}}(x) \vec{\sigma}_{\alpha \beta} \psi_{\beta}(x)
$$

where $\psi^{\mathrm{t}}(\psi)$ is the fermionic oscillator function and $\vec{\sigma}_{\alpha \beta}$ is the vector of Pauli matrices. A unit vector $\vec{n}$ is constructed as

$$
\begin{aligned}
& \vec{n}=\left(\psi_{1}^{*} \psi_{2}^{*}\right) \vec{\sigma}\left(\begin{array}{l}
\psi_{1} \\
\psi_{2}
\end{array}\right) \\
& \text { with } \psi_{1}=(\cos \theta / 2) e^{i \varphi / 2} \\
& \psi_{2}=(\sin \theta / 2) e^{-i \varphi / 2}
\end{aligned}
$$

This helps us to write 


$$
\vec{S}(x)=(\sqrt{3} /) \Psi_{\alpha}^{\dagger}(x) \vec{\sigma}_{\alpha \beta^{\psi} \beta}
$$

We can now construct a unit vector $n_{\mu}$ with $\mu=0,1,2,3$ in $3+1$ dimensions incorporating the unit vector $\vec{n}$ given by eqn. (2)

$$
n_{u}=(1 / \sqrt{2})\left(\psi_{1}^{*} \psi_{2}^{*}\right) \sigma_{\mu}\left(\begin{array}{l}
\psi_{1} \\
\psi_{2}
\end{array}\right)
$$

with $\sigma_{0}=\mathrm{I}$, I being the identity matrix and $\vec{\sigma}$ are Pauli matrices. We now construct the topological current

$$
J_{\mu}=\left(1 / 12 \pi^{2}\right) \varepsilon_{\mu \mathcal{A} \sigma} \varepsilon_{a b c d} n_{a} \partial_{V} n_{b} \partial_{\lambda} n_{c} \partial_{\sigma} n_{d}
$$

where a,b,c,d correspond to $(0,1,2,3)$ and $(\mu, \nu, \lambda, \sigma)$ correspond to space-time indices. The current $J_{\mu}$ can be written in the form ${ }^{11}$

$$
J_{\mu}=\left(1 / 24 \pi^{2}\right\}_{t}{ }_{\mu \mathcal{A} \sigma} \operatorname{VTr}\left(g^{-1} \partial_{V} g\right)\left(g^{-1} \partial_{\lambda} g\right)\left(g^{-1} \partial_{\sigma} g\right)
$$

with $g=n_{0} \mathrm{I}+i \vec{n} \cdot \vec{\sigma}$ which belongs to the group $S U(2)$. If we now demand that in Euclidean 4- dimensional space-time the field strength $F_{\mu \nu}$ of a gauge potential $A_{\mu}$ vanishes at all points on the boundary $\mathrm{S}^{3}$ of a certain volume $V^{4}$ inside which ${ }_{A_{\mu}=g^{-1} \partial_{\mu} g}$ the gauge potential tends to a pure gauge towards the boundary and we write

$$
A_{\mu}=g^{-1} \partial_{\mu} g
$$

with $g \varepsilon S U(2)$.

We can now write the topological current given by (7) as ${ }^{10}$

$$
J_{\mu}=\left(1 / 16 \pi^{2}\right) \varepsilon^{u v \lambda \sigma} \operatorname{Tr}\left\{A_{V} F_{\lambda \sigma}+(2 / 3) A_{V} A_{\lambda} A_{\sigma}\right\}
$$

With $A_{\mu}$ given by eqn. (8). It is noted that as the spin vector is constructed from the unit vector $J_{\mu}$ given by (2) which is incorporated in the current $J_{\mu}$ as is evident from eqn. (6), we can associate spin with this current $J_{\mu}$.

In fact we can consider the topological Lagrangian in terms of the $S U(2)$ gauge fields in affine spece

$$
L=-(1 / 4) \operatorname{Tr} \varepsilon^{\mu v \alpha \beta} F_{\mu v} F_{\alpha \beta}
$$

This gives rise to the topological current ${ }^{12}$

$$
\vec{J}_{\mu}=\varepsilon^{\mu \nu \lambda \sigma} \vec{a}_{V} \times \vec{f}_{\lambda \sigma}=\varepsilon^{\mu \nu \lambda \sigma} \partial_{V} \vec{f}_{\lambda \sigma}
$$

where we have taken the $S U(2)$ gauge field $A_{\mu}$ and corresponding field strength $F_{\mu v}$ as

$$
A_{\mu}=\vec{a}_{\mu} \cdot \vec{\sigma} \text { and } F_{\mu V}=\vec{f}_{\mu V} \cdot \vec{\sigma}
$$

$\vec{\sigma}$ being vector of Pauli matrices. From this it appears that the spin vector $\vec{S}(x)$ can be depicted as the topological current $\vec{J}_{\mu}$ given by eqn.(11). In terms ofthis current a spin system on a lattice can be viewed as if currents are located on the vertices when gauge fields lie on links. ${ }^{9}$ This helps us to consider the spin system associated with a DNA super coil in terms of the Chern- Simons topology as will be discussed in the next section.

\section{Topological properties of a DNA molecule}

In length scales of a large number of base pairs DNA in vivo is organized into topologically independent loops. The two strands of a circular DNA molecule possess as a topological invariant the number of times they wind around each other which is known as the linking number. A B-DNA molecule has one right-handed twist per $h=3.4 \mathrm{~nm}$ along its length. When these are closed in a planar circle without twisting of the ends the resulting linking number is $L k_{0}=L / h=\omega_{0} L /(2 \pi)$ where $L$ is the length and $\omega_{0}$ is the spatial rotation rate of the base pairs about the central axis. Deviations in the twisting rate from $\omega_{0}$ are measured relative to $L k_{o}$ through the parameter defining the excess linking $\sigma=\left(\Delta L k / L k_{0}\right)$ where $\Delta L k=L k-L k_{0}$. The linking number $L k$ is expressed as $L k=T w+W r$ where $T w$ represents the twist corresponding to the rotation of the internal degrees of freedom about the molecule axis and $W r$ represents the writhe. ${ }^{13,14}$ The twist measures the winding of one curve about the other .It can be mathematically expressed as ${ }^{6}$

$$
T w=\int_{0}^{L}(d s / 2 \pi)\left[\omega_{0}+\Omega(s)\right]=L k_{o}+\Delta T w
$$

where $\vec{r}(s)$ is the twist strain measuring the excess or deficit rotation of the base pairs about the axis and $S$ defines the arc length. The Writhe characterizes the chiral deformation of a curve. One can assign an orientation to a curve and compute the sum of signed crossings in a planar projection along every direction. $\vec{r}(s)$ is equal to the average of such sums over all projections ${ }^{15}$

For a configuration $\vec{r}(s)$ depicting a space curve parameterized by arc length $s$, we define the tangent vector $\vec{t}=\partial_{s} \vec{r}$ which traces out a closed path on the unit sphere. The writhe is equal to the total area $\mathrm{A}$ on the unit sphere enclosed by the path divided by $2 \pi .{ }^{16}$

$$
W r=\mathrm{A} / 2 \pi
$$

Right-handed and left -handed circulation on the sphere contribute positively and negatively respectively to $A$. The length of a molecule in $1 \mathrm{rad}$ of the superhelix is $l=\sqrt{R^{2}+P^{2}}$ where $R(P)$ represents the radius (pitch) of the superhelix, the helix repeat length being $2 \pi p$. The helix angle $\gamma$ is defined by $\sin \gamma=P / 1 . \gamma$ takes values between 0 and $\pi / 2$ where $\gamma=0(\pi / 2)$ corresponds to circle (straight line). A solenoidal superhelix represents a toroidal structure. For B-DNA a plectonemic superhelix consists of two right-handed helices that are interwound. At the end of the resulting cylindrical structure, the two helices are connected. The writhes of plectonemic and solonoidal coils are ${ }^{14}$

$$
W r=\left\{\begin{array}{l}
\mp 2 n \sin \gamma \text { (plectoneme) } \\
\pm n(1-\sin \gamma) \text { (solenoid) }
\end{array}\right.
$$

where $n$ is the number of superhelix repeats given by $n=L / 4 \pi l$ for the plectoneme and $n=L / 2 \pi l$ for the solenoid. The upper (lower) sign corresponds to the value for right (left- handed) super coil. From the point of view that a DNA super coil can be depicted as a spin system we can determine the linking number from the spin degrees of freedom. It is noted that the expression of the current $J_{\mu}$ associated with the spin given by eqn.(9) essentially corresponds to the ChernSimons secondary characteristics class. The topological charge

$$
q=\int J_{0} d^{3} x
$$

Corresponds to the winding number associated with the homotopy $\pi_{3}\left(S^{3}\right)=Z$ and can be written as

$$
q=2 \mu=\left(1 / 24 \pi^{2}\right) \int_{S^{3}} \varepsilon^{\mu \nu \mathcal{A} \sigma} \operatorname{Tr}\left(g^{-1} \partial_{V} g\right)\left(g^{-1} \partial_{\lambda} g\right)\left(g^{-1} \partial_{\sigma} g\right)
$$

This charge $q$ essentially represents the Pontryagin index which is an integer and the relation $q=2 \mu$ implies that $\mu$ corresponds to the magnetic monopole strength and can take the value

$$
\mu=0, \pm 1 / 2, \pm 1, \pm 3 / 2 \text {. }
$$

This Pontryagin index can be written as the integral in the 4-dimensional manifold $M_{4}$ as

$$
q=\int_{M_{4}} \operatorname{Tr}(F \wedge F)
$$


where $F$ is the two-form related to the field strength associated with the $S U(2)$ gauge field $A_{\mu}$. Now from the relation

$$
\int_{M_{4}} \operatorname{Tr}(F \wedge F)=\int_{M_{4}} \operatorname{Tr}(A \wedge d A+(2 / 3) A \wedge A \wedge A)
$$

where $M_{3}$ is a three dimensional manifold and $A$ is the one-form corresponding to the $S U(2)$ gauge field $J_{u}$ we note that the R.H.S of eqn.(19) represents the Chern-Simons invariant and is thus found to be associated with the Pontryagin index. Noting that the Pontryagin index corresponding to the charge related to the gauge current $J_{u}$ given by eqn.(9) which is associated with the spin, we can consider spin in the framework of Chern-Simons topology.In fact from eqn.(11) we note that any component of the spin vector can be written as

$$
J_{\mu}^{a}(a=1,2,3)=\varepsilon^{\mu \nu \lambda \sigma} a_{V} \partial_{\lambda} a_{\sigma}
$$

where $a_{V}$ corresponds to an Abelian gauge field. When we project it onto a three dimensional manifold this corresponds to the ChernSimons term $\varepsilon^{V} \lambda \sigma a_{V} \partial_{\lambda} a_{\sigma}$. In the Abelian theory we consider the one-form $a$ associated with the gauge field $a_{V}$ and choose the action

$$
S=(k / 8 \pi) \int_{M_{3}} \varepsilon^{l j k} a_{i} \partial_{j} a_{k}
$$

where $k$ is an integer. We now pick up some circles $C_{a}$ and some integers $n_{a}$ corresponding to representations of the Abelian gauge group. It is assumed that two curve $C_{a}$ and $c_{l}$ do not intersect for $a \neq b$. As shown by Polyakorv ${ }^{17}$ the expectation value of the product

$$
W=\prod \exp \left(\operatorname{in}_{a} \int_{C_{a}} a\right.
$$

with respect to the measure determined by $e^{i S}$ is given by

$$
\langle W\rangle=\left(\exp \left((\mathrm{i} / 2 k) \sum_{a, b} n_{a} n_{b} \int_{C_{a}} d x^{i} \int_{C_{b}} d y^{i} \varepsilon_{i j k}\left\{(x-y)^{\mathrm{k}} /|x-y|^{2}\right\}\right)\right.
$$

For $a \neq b$ this integral is essentially the linking number

$$
\varphi\left(C_{a}, C_{b}\right)=\frac{1}{4 / \pi} \int_{C_{a}} d x^{i} \int_{C_{b}} d y^{j} \varepsilon_{i j k}\left\{(x-y)^{\mathrm{k}} /|x-y|^{2}\right\}
$$

As long as $C_{a}$ and $C_{a}$ do not intersect $\varphi\left(C_{a}, C_{b}\right)$ is a well defined integer. Thus ignoring the term $a=b$, we have

$$
\langle W\rangle=\exp \left((2 \pi i / k) \sum_{a, b} n_{a} n_{b} \varphi\left(C_{a}, C_{b}\right)\right)
$$

The appearance of linking number from the Chern-Simons term associated with the gauge current representing the spin suggest that the linking number can be associated with a spin system.

From this analysis it appears that when a DNA super coil is represented as a spin system the linking number can be considered as a topological invariant related to the Chern-Simon topology associated with the spin system. It should be mentioned that though the linking number is a topological invariant when it is split into twist $(\mathrm{Wr})$ and writhe $(W r)$ these entities are not topological invariants. Since the linking number of a close DNA molecule remains constant during any deformation of the molecule that preserves chemical bonding, it can only be changed by mechanisms in which chemical bonds are disrupted..$^{15}$

\section{Elastic properties of DNA supercils}

Here we study the elastic properties of a DNA super coil from on analysis of the spin degrees of freedom. As spins are considered here as gauge currents constructed from $S U(2)$ gauge fields we map the elastic properties onto the space of gauge potentials. To this end we associate the unit tangent vector $\vec{t}=\partial_{s} \vec{r}(s)$ where $\vec{r}(s)$ depicts a space curve parameterized by arc length $S$ with the vector of gauge potentials $\vec{A}_{\mu}$ where the components correspond to the $S U(2)$ gauge fields $A_{\mu}^{a}(a=1,2,3)$ a being the group index and $\mu$ is the space time index. Evidently $\partial_{s}^{2} \vec{r}$ correspond to the $S U(2)$ gauge field strength $\vec{F}_{\mu V}$. In view of this the internal energy corresponding to the curvature (bending) given by $\int d s\left(\partial_{s}^{2} \vec{r}\right)^{2}$ can be written as $\int d^{3} x \operatorname{Tr} F_{\mu \nu} F_{\mu v}$ The twisting elastic energy can be associated with the spin-spin interaction and can be written in the continuum limit as $m^{2} \int \vec{J}_{\mu} \vec{J}_{\lambda} d^{3} x$. where $\vec{J}_{\mu}$ is the gauge current ${ }^{11}$ and $m$ is a constant having the dimension of mass. It should be mentioned that this term corresponding to the spinspin interaction in the continuum limit effectively represents torsion. ${ }^{18}$ The elastic energy of B-DNA of length $\mathrm{L}$ is given $\mathrm{by}^{6}$

$$
E_{c l} / k_{B} T=\frac{1}{2} \int_{0}^{L} d S\left[A\left(\partial_{s}^{2} \vec{r}\right)^{2}+C \Omega^{2}\right]
$$

where the deviation in the twisting rate from $\mathrm{Co}_{0}$ is described by the scalar field $\Omega(s), A(C)$ being the bending (twisting) elastic constant. When we map this on the configuration space of gauge potentials associated with the spin system, we can write for the elastic energy in the continuum limit.

$$
E_{c l} / k_{B} T=\frac{1}{2} \int d^{3} x\left[A T r F_{\mu v} F_{\mu v}+C m 2 \vec{J}_{\mu} \vec{J}_{\mu}\right]
$$

where $\vec{J}_{\mu}$ is given by eqn.(11). A significant result of this analysis is that curvature (bending) and torsion (twisting) associated with the deviation from $\omega_{0}$ is not separate entities. Indeed one is related to the other. This follows from the fact that the gauge field curvature $F_{\mu \nu}$ is incorporated in the construction of the current $\vec{J}_{\mu}$ in eqn. (11) which generates torsion. Indeed this interesting relationship between bending and torsion has been pointed out by Nelson ${ }^{19}$ suggesting that intrinsic bending can have a huge effect in the transport of torsional stress along DNA.

It may be mentioned that twisting is measured by the spatial rotation rate of base pairs about the central axis which for an undistorted DNA is just $\mathrm{Co}_{0}$. However for a distorted DNA twisting elastic energy is non-zero when the double-helix twist is altered from $c o_{0}$ In this case we have chiral symmetry breaking which is manifested through torsion. It is noted that in the present formalism torsion is generated by the topological current $J_{\mu}^{a}(a=1,2,3)$ which is related to the axial vector current $J_{\mu}^{5}=\bar{\psi} \gamma_{\mu} \gamma_{5} \psi$ associated with a chiral spinor.In fact each component of the vector $\vec{J}_{\mu}$ in eqn. (11) is related to the axial vector current through the relation ${ }^{20}$

$$
\partial_{\mu} J_{\mu}^{2}=-1 / 2 \partial_{\partial_{\mu}} J_{\mu}^{5}=-1 / 2 J_{\mu}^{2}, J_{\mu}^{3}=1 / 2 J_{\mu}^{2}
$$

In quantum field theory the divergence of this current is nonzero which gives rise to chiral anomaly caused by chiral symmetry breaking. In view of this we find that twisting elastic energy represented by torsion has a correspondence with the chiral anomaly in quantum field theory.

\section{DNA Super coil and Enyanglement Entropy}

An interwound plectonemic super coil consists of two helices of the same handedness and at the end of the cylindrical structure the two helices are connected. A solenoidal super coil is closed by slow distortion of the coil into a toroidal structure. Thermal fluctuation swells up the super coil radius to larger than the super coil hard core radius. In fact it has been shown that a repulsive effective entropic potential arises opposing the elastically driven collapse at zero temperature. ${ }^{6}$ It implies that thermodynamic entropy plays a significant role in a DNA super coil. When DNA molecules are treated as spin systems, it can be shown that super coils attain the 
entanglement entropy due to the entanglement of spins. In fact in a super coil we can consider that the spins associated with each DNA loop are arranged along the axis of the super coil. As we have pointed out that in a DNA molecule spins are considered to be arranged in an antiferromagnetic chain, the super coil axis may be treated as a lattice of antiferromagnetic spin system. To have the minimal energy two adjacent spins of opposite orientations will form a singlet. Due to chirality caused by twisting strain into the loop related to torsion the spin system will be in a frustrated state as frustration leads to chirality. This frustration suggests that spin singlets are formed by resonating valence bond (RVB). ${ }^{21}$

It has been shown in some earlier papers ${ }^{22,23}$ that the measure of the entanglement of formation given by concurrence $C$ for the entanglement of a pair of nearest neighbour spins is related to the Berry phase $e^{24}$ given by $e^{i \varphi_{B}}=e^{i 2 \pi \varphi_{B}}$ which is acquired by a spin state when the spins in the system are rotated about the quantization axis (Z-axis) in a closed circuit. In fact we have the relation

$$
C=\left|\tilde{\varphi}_{B}\right|=\left|\varphi_{B}\right| / 2 \pi
$$

It has been observed that the concurrence associated with the entanglement of formation for a pair of nearest neighbour spins in a frustrated system is given by $C=0.5^{22}$. Thus the total concurrence accumulated in the spin chain is given by

$$
C=\sum_{i=1}^{L / 2}\left|\tilde{\varphi}_{B}\right|=\left|\tilde{\varphi}_{B}\right|(L / 2)=(1 / 2)(L / 2)=L / 4
$$

where $L$ is the total number of spins in the chain and $L / 2$ is the number of singlets. It is noted that the von Neumann entropy for an entangled spin system in a pure state is reduced to the entanglement of formation given by concurrence in a mixed state..$^{25}$ Thus the above expression (30) represents the entanglement entropy of the spin system. Now we consider that the super coiled stiff polymer (DNA) is confined inside a narrow tube of radius $r$. The area of a DNA loop in a super coil is determined by the number of coils in the loop and hence by the number of spins in the loop. When the super coiled stiff polymer is confined in a narrow tube of radius $r$ we can associate the area of the surface of the tube with the number of spins in the DNA loop at that surface. Now from the holographic principle which states that for closed surface entropy is given by the area of the surface the entanglement entropy is found to be given by the area of the surface of the tube. Thus the entanglement entropy can be written in the form

$$
S \approx r^{2} / 4 r_{0}^{2}
$$

$r$ being the radius of the tube and $r_{0}$ a fundamental area unit. If we identify $r \approx 2 A$ where $A$ is the bending elastic constant and $2 A$ corresponds to the step length of an equivalent flexible polymer and $r_{0}$ is taken to represent the radial displacement of a given point on the coil which is of the order of $R .^{6} \mathrm{We}$ find the entanglement entropy is given by

$$
S \approx A^{2} / R^{2}
$$

Similarly for the displacement of a given point on the coil along the superciol axis which is of the order of $\pi P$

$$
S \approx A^{2} /(\pi P)^{2}
$$

So the total entropy is given by

$$
S=A^{2} / R^{2}+A^{2} /(\pi P)^{2}
$$

Now we observe that this entanglement entropy effectively corresponds to the thermodynamic entropy. Indeed for a tube of narrow radius entanglement entropy cannot vanish whereas in the limiting case of radius $r \rightarrow 0$ we can think of zero radius (straight line) when the total elastic energy vanishes at zero temperature. In this case the entanglement entropy also vanishes. In fact at zero temperature an elastic tube will collapse into a plectonemic super coil when subject to the constraint $\Delta L k / L \neq 0 .{ }^{26}$ When $\Delta L k$ is put into writhe $(W r)$ the twist energy becomes zero. Then we can make the plectoneme collapsed into a line $(\operatorname{Sin} \gamma=1)$ which makes the bending energy zero also. Now at zero temperature the area of the loop vanishes. In this case the entanglement entropy also vanishes. In view of this we can identify the entanglement entropy as the thermodynamic entropy of the super coil.

This helps us to compute the free energy associated with the super coil. We note that from the relation $d F=S d T, F(T)$ being the free energy (temperature), we note that the free energy per unit volume associated with the entropy given by eqn. (34) can be written as

$$
\Delta F /\left(K_{B} T V\right) \approx A^{2} /\left(R^{2} r^{3}\right)+A^{2} /\left\{(\pi P)^{2} r^{3}\right\}
$$

This gives the free energy per unit length

$$
\Delta F /\left(K_{B} T L\right) \approx A^{2 / 3} /\left(R^{2 / 3} r\right)+A^{2 / 3} /\left\{(\pi P)^{2} r^{3}\right\}
$$

Now as argued above $r$ is taken to be of the order of $A$ and hence we find

$$
\begin{aligned}
\Delta F /\left(K_{B} T L\right) & \approx A^{2 / 3} /\left(R^{2 / 3} A\right)+A^{2 / 3} /\left\{(\pi P)^{2} A\right\} \\
& =1 /\left(A^{1 / 3} R^{2 / 3}\right)+1 /\left(A^{1 / 3}(\pi P)^{2 / 3}\right)
\end{aligned}
$$

This result is identical with that obtained by Marko et al. ${ }^{6}$

A crucial implication of our result is that as entanglement of a quantum system is the major resource in processing quantum information, ${ }^{27,28}$ the entanglement entropy determines the quantity of information which can be used for transcription. When DNA is regarded as linear repository of sequence information we note that this entanglement entropy determines the quantity of genetic information in a super coil which can be transcribed.

\section{DNA Loop as a Soliton}

A DNA molecule is characterized by certain topological feature such as linking number. At length scale of thousands of base pairs DNA is organized into topologically independent loops. There are situations in vivo when topological constraints lead to super coiling. DNA loops in a super coil may behave as a topological object such as a soliton (skyrmion) which is realized when we consider DNA as a spin system. In fact DNA loops in a super coil when constrained by a change in the linking number due to deviation of twisting rate from $\omega_{0}$ correspond to the formation of a spin texture when a DNA molecule is considered as a spin system. A change in the linking number from $L k_{o}$ due to twist of the ends causing a deviation from the planar circle configuration corresponds to a spin texture and represents a deviation of the spin system from the ground state when spin excitations occur. These excitations resemble the solitons (skyrmions) described by the nonlinear $\sigma$-model. As mentioned in sec. 2 a spin may be depicted in terms of fermionic oscillators. We can depict a two-component spinor as $\begin{aligned} {\left[\begin{array}{l}u \\ v\end{array}\right] } & \text { with } \\ u & =\cos \theta / 2 e^{i \varphi} / 2 \\ v & =\sin \theta / 2 e^{-i \varphi} / 2\end{aligned}$

In terms of the spin system we can consider the ground state wave 
function depicting the DNA super coil with linking number $L k_{o}$

$$
\left|\psi_{0}\right\rangle=\prod_{i\langle J}\left(u_{i} v_{j}-v_{i} u_{j}\right)
$$

where $i$ and $j$ correspond to the spin sites. When the linking number deviates from $L k_{o}$ due to deviation of the twisting rate from $c o_{0}$, the resulting skyrmion state is described by

$$
|\psi\rangle=C \prod_{k}\left(\begin{array}{l}
v_{k} \\
-\alpha u_{k}
\end{array}\right)\left|\psi_{0}\right\rangle
$$

where the spin texture is included within the components $v_{k}$, $u_{k}$ and $0 \leq \alpha \leq 1 .{ }^{29}$ If a smooth and monotonical function $g(\theta)$ is defined with $g(0)=0$ and $g(\pi)=\pi$ then the skyrmion state can be written as

$$
\vec{\varphi}(\Omega)=\cos (g(\theta)-\theta) \vec{e}_{r}+\sin (g(\theta)-\theta) \vec{e}_{\theta}
$$

where $\vec{e}_{r}$ and $\vec{e}_{r}$ are the basis vectors. The size of a skyrmion is determined by the function $g(\theta)$ and $g(\theta)=\theta$ describes the hedgehog skyrmion with spin in the radial direction $\vec{r}$. The skyrmion state $\vec{\varphi}(\Omega)$ is constrained by the relation $|\vec{\varphi}(\Omega)|=1$. The quantum state for the skyrmion $\vec{\varphi}(\Omega)$ can be written as

$$
|\psi\rangle=C \prod_{k}\left(\begin{array}{c}
\operatorname{Sin}^{g\left(\theta_{k}\right)} / 2 e^{-i \varphi_{k}} / 2 \\
\operatorname{Cos}^{g\left(\theta_{k}\right)} / 2 e^{i \varphi_{k}} / 2
\end{array}\right)\left|\psi_{0}\right\rangle
$$

where $C$ is the normalization constant and $g(\theta)$ controls the size of the skyrmion. From eqn.(41) and (42) it is seen that $0 \leq \alpha \leq 1$ is determined from $g(\theta)$ and $\alpha$ controls the size of the skyrmion. Indeed we can define

$$
\theta=2 \arctan \alpha
$$

which equals $\pi / 2$ for the hedgehog skyrmion with $\alpha=1$.

Taking the spin variable $\vec{z}=U \vec{z}_{0}$ with $\vec{z}_{o}=\left(\begin{array}{l}1 \\ 0\end{array}\right)$ and $U \varepsilon S U(2)$ we may write the nonlinear $\sigma$-model Lagrangian in terms of the $S U(2)$ matrices $U$ as ${ }^{30}$

$$
L=-\left(M^{2} / 16\right)\left(\partial_{u} U^{\dagger} \partial_{\mu} U\right)-\left(1 / 32 \eta^{2}\right)\left(\partial_{\mu} U U^{\dagger}, \partial_{V} U U^{\dagger}\right)^{2}
$$

where $M$ is a constant having dimension of mass and $\eta$ is a dimensionless parameter, $\mu, v$ being space -time indices. The $\alpha$ dependence may be incorporated through $M$ and $\eta$ where these parameters are taken as functions of $\alpha$.

For a distorted loop we can consider the radius of the loop $R$ as a function, $R(\theta, \varphi)$ corresponding to the core radius of the skyrmion. We can define the core size of the skyrmion such that $R=R_{0}(1-\alpha)$ where $R_{0}$ is the size of the skyrmion with minimal energy. The static nonlinear $\sigma$-model Lagrangian corresponding to eqn. (44) gives rise to the energy integral as

$$
E=\int d^{3} x\left\{\left(M^{2} / 16\right) \operatorname{Tr}\left(\nabla U^{\dagger} \nabla U\right)+\left(1 / 32 \eta^{2}\right) \operatorname{Tr}\left[\partial_{i} U U^{\dagger}, \partial_{j} U U^{\dagger}\right]^{2}\right\}
$$

where $i, j=1,2,3$ are spacial indices. To compute the energy we take the Skyrme ansatz

$$
U(x)=\exp (i F(r) \vec{\tau} \cdot \hat{x}
$$

where $\vec{\tau}$ are Pauli matrices, $\hat{x}=\frac{\vec{x}}{r}$ and $F(\mathrm{O})=\pi$ and $F(r) \rightarrow 0$ as $r \rightarrow \infty$. We explicitly write

$$
\begin{aligned}
& U=\operatorname{Cos} F(r)+i \vec{\tau} \cdot \hat{x} \operatorname{Sin} F(r) \\
& \text { with } \\
& \cos F(r)=(1-r / R)^{2} /(1+r / R)^{2} \text { and } \\
& \sin F(r)=2(r / R)^{2} / 1+(r / R)^{2}
\end{aligned}
$$

The energy integral becomes

$$
E(R)=4 \pi^{2} M^{2} R I_{1}+2 \pi^{2}\left(I_{2} / \eta^{2} R\right)
$$

where

$I_{1}=\frac{1}{\pi} \int_{0}^{\alpha} d x\left[\sin ^{2} F(r)+x^{2}(\partial F / \partial x)^{2}\right]=3.0$

and

$I_{2}=(1 / \pi) \int_{0}^{\alpha} d x\left[\left(\sin ^{4} F(r) / x^{2}\right)+\sin ^{2} F(r)(\partial F / \partial x)^{2}\right]=1.5$

with $x^{0}=r / R$. This gives the expression of energy

$$
E(R)=12 \pi^{2} M^{2} R+\left(3 \pi^{2} / \eta^{2} R\right)
$$

The minimum of energy $E(R)$ is found from the relation

$$
\partial E(R) / \partial R=12 \pi^{2} M^{2}-3 \pi^{2} / \eta^{2} R^{2}=0
$$

which gives for $E_{\min }$ the size as

$$
R_{0}=1 / 2 M \eta
$$

and the energy

$$
E_{\min }=E\left(\mathrm{R}_{0}\right)=12 \pi^{2} M / \eta
$$

It is noted that the coupling parameters $M$ and $M$ are functions of $\alpha$ such that in the limit $\alpha \rightarrow 0, M(\alpha) \rightarrow 0$ and $\eta(\alpha) \rightarrow 0$ but $M / \eta$ is fixed. When we take $R=R_{0}(1-\alpha)$ we have

$$
E(R)=\left\{\left(6 \pi^{2} M\right) / \eta\right\}[(1-\alpha)+1 /(1-\alpha)]
$$

Now we note that the parameter $\alpha$ effectively gives a measure of the chirality associated with twisting strain into the loop given by $\sigma=\left(\Delta L k / L k_{0}\right)$. In fact in the simplest form we can take $\alpha=k|\sigma|$ where $k$ is a constant. So form the relation $R=R_{0}(1-\alpha)=R_{0}(1-k|\sigma|)$ , we can estimate the energy of a DNA loop as a function of $\sigma$. It is noted that the relation $R=R_{0}(1-\alpha)$ gives a nonzero size for $\alpha=1(\sigma=0)$ when $R_{0}$ is infinite. Indeed it has been found that for $|\sigma|<0.02$ the minimal free energy state has $R=P=\infty$ indicating that no consistent stable super coiled state exists for small $|\sigma|$. For $|\sigma|>0.02$ the plectonemic free energy exhibits a minimum value for finite $R$ and $P$ which implies that we have a stable super coiled state. It appears that $\sigma$ can be varied through roughly -0.1 to 0.1 as beyond these bounds the double helix is unstable. ${ }^{31}$ These observations are found to be consistent with this skyrmion model.

In Figure 1 we have plotted the radius $R$ of a plectonemic super coil as a function of $\sigma$ where $R$ is given by $R=R_{0}(1-\alpha)$ with $\alpha=k|\sigma|$. The constant $k$ is determined from the experimental data ${ }^{7}$ and the best fit is given by $k=8.333$. In Figure 2 we have plotted the Skyrmion energy as a function of radius $\mathrm{R}$.

From our analysis it appears that when the long linear chromosomal DNA molecules are organized into loops, these topological independent loops appear as solitons. Solitons are nonlinear excitations which can travel as coherent solitary waves. The present analysis implies that soliton excitations may well exist in DNA chains which is consistent with the observations of Englander et.al. ${ }^{32}$ The linking number associated with a super coil is given by the topological charge of the loops. As the skyrmion (soliton) depicting a loop is described by the nonlinear $\sigma$-model in terms of $S U(2)$ gauge fields, the topological charge of a loop is given by the winding number of the mapping of the 3-space manifold into the group manifold $S U(2)=S^{3}$ which corresponds to homotopy $\pi_{3}(S U(2))=\pi_{3}\left(S^{3}\right)=Z$ where $Z$ represents the set of integers. When DNA loops super coil the linking 
number is given by an integer determined by this homotopy group so that $L k=n Z$ where $n$ is the number of superhelix is loops.

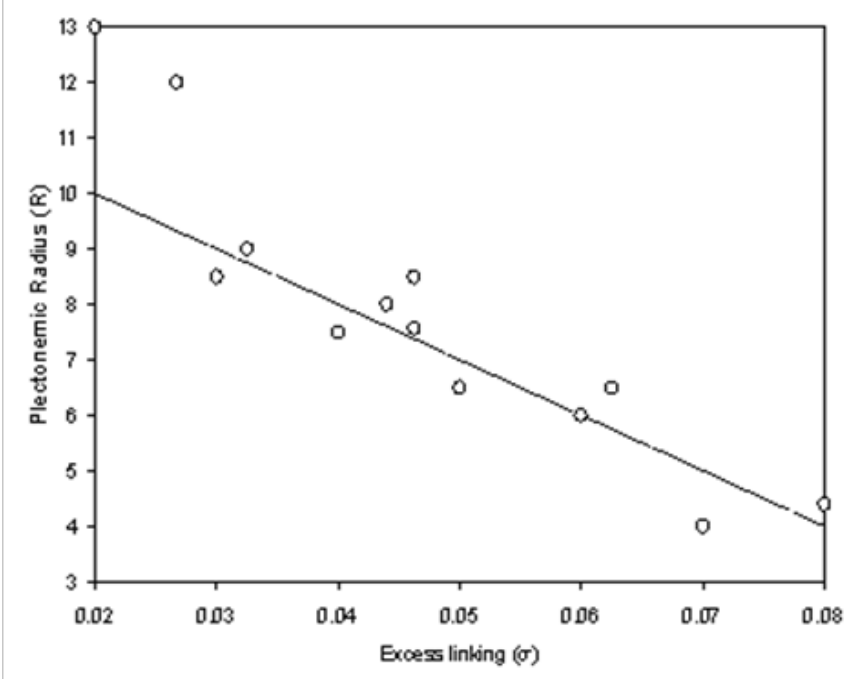

Figure I Radius $R$ of a plectonemic supercoil as a function of $\sigma$ where $R$ is given by (I ) $R=R_{0}(1-\alpha)$ with $\alpha=k|\sigma| \cdot$ The constant $\mathrm{k}$ is determined from the experimental data ${ }^{7}$ and the best fit is $\mathrm{c}$ given by $\mathrm{k}=8.333$.

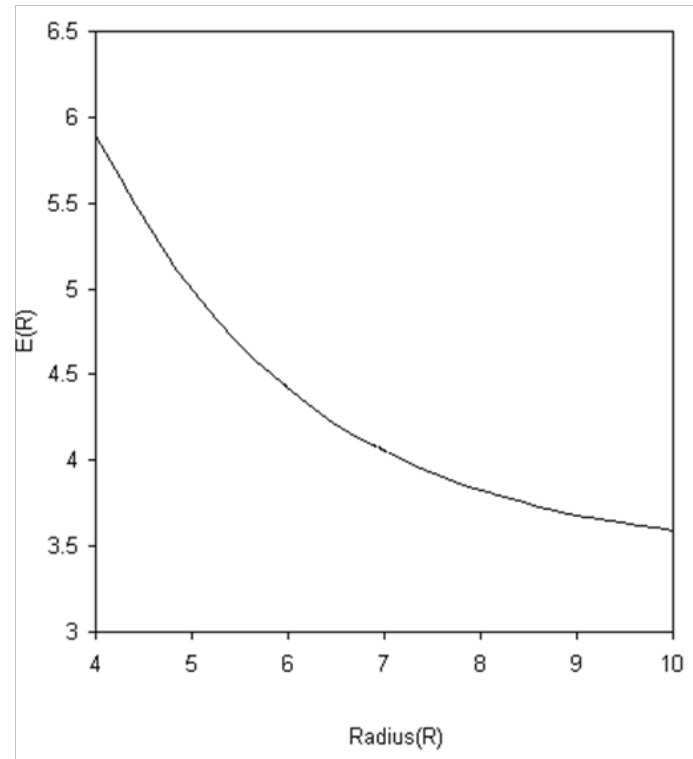

Figure 2 Skyrmion energy as a function of radius $\mathrm{R}$.

\section{Discussion}

We have shown that a DNA super coil can be treated as a quantum spin system such that spins are located on the axis forming an antiferromagnetic chain. These spins can be associated with $S U$ (2) gauge field currents when gauge fields lie on the links. We have formulated bending (curvature) and twisting (torsion) in terms of these gauge fields. A significant result of this formalism is that bending and twisting are not independent entities. In fact bending influences the propagation of twisting strain along the DNA which has been supported by experiments.

The formulation of DNA super coil in terms of an antiferromagnetic spin chain gives rise to the entanglement entropy which induces the entropic protential associated with the free energy per length corresponding to the entropy cost of confining a stiff polymer inside a narrow tube. The entanglement entropy effectively represents the thermodynamic entropy and this repulsive entropic potential opposes the elastically driven collapse of a super coil which can occur at zero temperature. The entanglement entropy has a very significant implication in that it measures the total information content in the system. Indeed when a DNA is regarded as a linear repository of sequence information the entanglement can be used to transcribe information and entropy determines the quantity of information which is available for transcription. In view of this a measure of this entanglement entropy can be taken to determine the quantity of genetic information which can be transcribed. This helps us to consider genetic information transcription as a manifestation of quantum information theory.

Another interesting aspect of our analysis is that a DNA loop can be treated as topological object depicted as a skyrmion (soliton) which arises due to the excitation of spins caused by the deviation of the twisting rate from $\mathrm{co}_{0}$ leading to excess (deficit) of linking number. The spin texture is determined by the twist parameterized by the quantity $\sigma=\left(\Delta L k / L k_{C}\right)$. The energy of the skyromion depicting a DNA loop depends on the radius which is determined by the parameter $\sigma$. The linking number of a DNA molecule when organized into loops is related to the topological charge of a skyrmion depicting a loop.

\section{Conclusion}

We have shown that a DNA super coil can be treated as a quantum spin system such that spins are located on the axis forming an antiferromagnetic chain .These spins can be associated with $S U$ (2) gauge field currents when gauge fields lie on the links. We have formulated bending (curvature) and twisting (torsion) in terms of these gauge fields. A significant result of this formalism is that bending and twisting are not independent entities. In fact bending influences the propagation of twisting strain along the DNA which has been supported by experiments.

The formulation of DNA super coil in terms of an antiferromagnetic spin chain gives rise to the entanglement entropy which induces the entropic protential associated with the free energy per length corresponding to the entropy cost of confining a stiff polymer inside a narrow tube. The entanglement entropy effectively represents the thermodynamic entropy and this repulsive entropic potential opposes the elastically driven collapse of a super coil which can occur at zero temperature. The entanglement entropy has a very significant implication in that it measures the total information content in the system. Indeed when a DNA is regarded as a linear repository of sequence information the entanglement can be used to transcribe information and entropy determines the quantity of information which is available for transcription. In view of this a measure of this entanglement entropy can be taken to determine the quantity of genetic information which can be transcribed. This helps us to consider genetic information transcription as a manifestation of quantum information theory.

Another interesting aspect of our analysis is that a DNA loop can be treated as topological object depicted as a skyrmion (soliton) which arises due to the excitation of spins caused by the deviation of the twisting rate from $\omega_{0}$ leading to excess (deficit) of linking number. The spin texture is determined by the twist parameterized by the quantity 
$\sigma=\left(\Delta L k / L k_{0}\right)$. The energy of the skyromion depicting a DNA loop depends on the radius which is determined by the parameter $\sigma$. The linking number of a DNA molecule when organized into loops is related to the topological charge of a skyrmion depicting a loop.

\section{Acknowledgments}

The authors are grateful to Sir Pratul Bandyopadhyay for helpful discussions.

\section{Conflicts of interest}

Authors declare there is no conflicts of interest.

\section{References}

1. A Worcel, EBurgi. On the structure of the folded chromosome of Escherichia coli. J Mol Biol. 1972;71(2):127-47.

2. C Benyajati, A Worcel. Isolation, characterization, and structure of the folded interphase genome of Drosophila melanogaster. Cell. 1976:393-407.

3. DA Jackson, P Dickinson, P R Cook. The size of chromatin loops in HeLa cells. EMBO J. 1990:567-571.

4. AP Wolffe. Chromatin. Academic New York. 1993.

5. LA Freeman, WT Garrard. DNA supercoiling in chromatin structure and gene expression. Crit Rev Eukaryot Gene Expr. 1992;2(2):165-209.

6. JF Marko, FD Siggia. Statistical mechanics of supercoiled DNA. Phys Rev E. 1990;52:2912.

7. TC Boles, JH White, NR Cozzarelli. Structure of plectonemically supercoiled DNA. J Mol Biol. 1990;213:931-951.

8. J Bednar, P Furrer, A Stasrak, et al. The twist, writhe and overall shape of supercoiled DNA change during counterion-induced transition from a loosely to a tightly interwound superhelix. Possible implications for DNA structure in vivo. J Mol Biol. 1994;235(3):825-847.

9. G Goswami, P.Bandyopadhyay. Spin system, gauge theory, and renormalization group equation. J Math Phys. 1995;34:749.

10. P Bandyopadhyay. The geometric phase and the spin-statistics relation. Proc Roy Soc A. 2010;466:2917.

11. AI Abanov, PB Wiegmann. Theta-terms in nonlinear sigma-models. Nucl Phys B. 2000;570(3):685-698.

12. M Carmeli, S Malin. Reformulation of general relativity as a gauge theory. Ann Phys. 1997;103(1):208-232.

13. I Calugareau, Crechoslovak. Sur les classes d'isotopie des noeuds tridimensionnels et leurs invariants. Math J. 1961;11(4):588-625.
14. J H White. Self-Linking and the Gauss Integral in Higher Dimensions. Am J Math. 1969;91:693-728.

15. D Swigon. Mathematics of DNA Structure,Function and Interactions. In Benham CJ, Harvey S, Olson WK, et.al editors. New York: Springer Science Business Media; 2009. p. 335.

16. FB Fuller. Decomposition of the linking number of a closed ribbon: A problem from molecular biology. Proc Natl Acad Sci USA. 1978;75(8):3557-3561.

17. AM Polyakov. Fermi-Bose Transmutations Induced By Gauge Fields Mod Phys Lett A. 1988;3(3)325-328.

18. A Bandyopadhyay, P Chatterjee, P Bandyopadhyay. SL(2,C)-gauge theory,N=1 supergravity and torsion. Gen Rel Grav. 1986;18:1193-1205.

19. P Nelson. Transport of torsional stress in DNA. Proc Natl Acad Sci U S A. 1999;96(25):14342-14347.

20. A Roy, P Bandyopadhyay. Topological aspects of a fermion and the chiral anomaly. J Math Phys. 1989;30(10):2366.

21. PW Anderson. The Resonating Valence Bond State in $\mathrm{La}_{2} \mathrm{CuO}_{4}$ and Superconductivity. Science. 1987;235:1196-1198.

22. B Basu, P Bandyopadhyay. A geometrical approach towards entanglement. Int J Geo Meth Mod Phys. 2007;9:707-716.

23. B Basu, P Bandyopadhyay. Spin entanglement of two delocalized Fermions and Berry phase. J Phys A. 2008;41:055301.

24. MV Berry. Quantal phase factors accompanying adiabatic changes. Proc Roy Soc A. 1984;392:45.

25. TJ Osborne, MA Nielson. Entanglement in a simple quantum phase transition. Phys Rev A. 2002;66:032110.

26. NG Hunt, JF Hearst. Elastic model of DNA supercoiling in the infinitelength limit. J Chem Phys. 1991;95:9329.

27. CH Bennett, DP Divincenzo. Quantum information and computation. Nature. 2002;404:247-255.

28. MA Nelson, GLChuang. Quantum Information and Quantum Compoutation. UK: Cambridge University Press; 2000. p. 704.

29. B Basu, S Dhar, P Bandyopadhyay. Quantum hall skyrmions in the framework of $\mathrm{O}(4)$ nonlinear sigma model. Int $J$ Mod Phys. 2004;18(2):171-184.

30. THR Skyrme. A unified field theory of mesons and baryons. Nucl Phys. $1962 ; 31: 556-569$

31. NR Cozzarelli, T C Boles, J.White. DNA Topology and its Biological Effects. New York: Cold Spring Harbor Laboratory; 1990. p. 480.

32. SW Englander, NR Kallenbach, AJ Heeger, et al. Nature of the open state in long polynucleotide double helices: possibility of soliton excitations. Proc Natl Acad Sci USA. 1980;77(12):7222-7226. 